\title{
Photosynthetic electron-transfer reactions in the gametophyte of Pteris multifida reveal the presence of allelopathic interference from the invasive plant species Bidens pilosa
}
Kai-Mei Zhang ${ }^{\mathrm{a}, \mathrm{b}, \mathrm{c}, \mathrm{1}}$, Yu Shen ${ }^{\mathrm{d}, \mathrm{b}, 1}$, Xiao-Qi Zhou ${ }^{\mathrm{e}}$, Yan-Ming Fang ${ }^{\mathrm{a}, \mathrm{b}^{*}}$, Ying Liu ${ }^{\mathrm{a}, \mathrm{b}}$, Lena Q $\mathrm{Ma}^{\mathrm{f}, \mathrm{g}}$

a Co-Innovation Center for Sustainable Forestry in Southern China, Nanjing Forestry University, Nanjing, Jiangsu, 210037, China

b College of Biology and the Environment, Nanjing Forestry University, Nanjing, Jiangsu,210037, China

c Department of Botany, Smithsonian Institution, Washington, DC, 20013, USA

d College of Resources and Environmental Sciences, Nanjing Agricultural University, Nanjing, Jiangsu, 210095, China

e Environmental Futures Research Institute, Griffith University, Nathan, Brisbane, 4111, Australia

f State Key Laboratory of Pollution Control and Resource Reuse, School of the Environment, Nanjing University, Nanjing, Jiangsu 210046, China

g Soil and Water Science Department, University of Florida, Gainesville, FL 32611, USA

${ }^{1}$ These authors contributed equally to this work.

* Corresponding author. Tel.: +86 025-85427428; fax: +86 025-85427428.

E-mail address: jwu4@njfu.edu.cn (Y. M. Fang). 


\section{Abstract}

To date, the response of the fern gametophyte to its environment has received considerable attention. However, studies on the influence of plant invasion on the fern gametophyte are fewer. Allelopathy has been hypothesized to play an important role in biological invasion. Hence, it is necessary to study the allelopathy of invasive plant species to the fern gametophyte and elucidate the mechanisms by which invasive plants cause phytotoxicity. As one of the main invasive plants in China, Bidens pilosa exhibits allelopathic effects on the gametophytic growth of Pteris multifida. The root exudate plays an important role among various allelochemical delivery mechanisms in B. pilosa. The effect invasive plant species has on photosynthesis in native species is poorly understood. To elucidate this effect, the changes in photosynthesis in the gametophytes of P. multifida are analyzed to examine the mechanisms of the root exudates of B. pilosa. Meanwhile, a non-invasive plant, Coreopsis basalis, was also applied to investigate the effects on fluorescence and pigments in P. multifida gametophytes. We found that gametophytes exposed to both B. pilosa and C. basalis had decreased fluorescence parameters in comparison with the control, except for non-photochemical quenching. Furthermore, it was found that these parameters were markedly affected from day 2 to day 10 in the presence of both exudates at a concentration of $25 \%$ or above. B. pilosa exudate had a negative dose-dependent effect on chlorophyll $a$, chlorophyll $b$, carotenoid, and the total chlorophyll in the gametophyte. The inhibitory effects increased with increasing exudate concentrations of both species, exhibiting the greatest inhibition at day 10. In conclusion, B. pilosa irreversibly affected the photosynthesis of P. multifida on both PS I and PS II. Root exudates caused the primary damage with respect to the decrease of the acceptors and donors of photon and electron in photosynthetic units and the production and the relative yield of photochemical quantum in PS II. With the effects of exudates, part of the energy is released as heat in chloroplasts. The comparison of invasive and non-invasive plants in allelopathic experiments demonstrated that invasive plants were responsible for the critical damage to the photosynthetic process in local species. 
Keywords: Pteris multifida; gametophyte; Bidens pilosa; Coreopsis basalis; photosynthesis; allelopathy

Abbreviations: PSII, quantum yield of photosystem II; Fv/Fm, lower quantum efficiency of open PSII reaction centers; qP, photochemical fluorescence quenching; ETR, electron transport rate; NPQ, non-photochemical quenching; Chl $a$, Chlorophyll $a$; Chlorophyll $b$, Chl $b$; Car, carotenoid. 


\section{Introduction}

In recent years, biological invasion has become a phenomenon of environmental change with the increasing influence of human activity on the environment, and as such has become recognized as a major threat to the economy and environment worldwide because of its ecological impacts on ecosystems (Vitousek et al. 1996; Parker et al. 1999; Pimentel et al. 2005). In 2012, according to the preliminary statistics supplied by Ministry of Agriculture of PRC, the number of invasive species was over 448 in China, with a direct economic loss of about 20 billion dollars every year.

Invasive plant species can adversely affect the surrounding vegetation through allelopathic effects (Bais et al., 2003; Zhang et al., 2007, 2008a, 2008b). Bidens pilosa is one of the main invasive plants found in China. It can reduce agricultural production and alter plant community structures (Hao et al., 2009). Most studies on $B$. pilosa have focused on its deleterious effects on spermatophytes. It excretes allelochemicals and delivers them into the rhizosphere through leaching from the leaves and other aerial plant parts via different pathways that include volatile emissions, root exudate, and leaf litter. These allelochemicals hinder the germination and seedling growth of potential competitors (Khanh et al., 2009).

Ferns are the oldest extant vascular plants and the second-most diverse lineage of tracheophytes next to angiosperms ( $\mathrm{Lu}$ et al. 2015). An important aspect separating ferns from seed plants is that ferns possess an independent haploid gametophyte stage. Otherwise, in comparison with the gametophytes of angiosperms and gymnosperms the fern gametophyte is a photosynthetic free-living entity that is often described as a small, simple, delicate, ephemeral stage of the fern life cycle. Fern gametophytes have unique advantages as a model in investigating response to environment and revealing the mechanism compared to sporophytes (de Groot et al. 2011).

So far, the effects of invasive plants on ferns have received less attention. As such, invasive plants have threatened the growth and development of fern gametophytes. 
Hence, it is important to determine the allelopathic effects of invasive plants on fern gametophytes. In our previous work, we found that the cell membranes, antioxidant enzyme activities, and photosynthesis pigment contents of the gametophytes of the local fern species $P$. multifida were affected by leaf leachates of B. pilosa (Zhang et al. 2015). The results also showed that root exudates of $B$. pilosa adversely affected gametophytic survival, length of the rhizoid and biomass of gametophytes, and the conversion rate from gametophytic generation to sporophytic generation of $P$. multifida (Liu et al. 2015). Although (based on the evidence) B. pilosa is allelopathic to gametophytes of $P$. multifida, attention has been focused on determining secondary physiological processes such as germination, seedling growth, and the biomass of test species for most bioassays to assess the level of phytotoxic activity.

Environmental stress affects plant physiology at the whole plant and cellular levels (Ashraf and Foolad, 2007). However, the determination of other physiological and primary processes such as photosynthesis in native species affected by invasive species is still poorly understood (Aurélie et al., 2011). Photosynthesis inhibition is one of the best-characterized phytotoxic mechanisms induced by allelochemicals (Lorenzo et al., 2011; Hussain and Reigosa, 2011a). Root exudation plays an important role among the various mechanisms by which allelochemicals are delivered in B. pilosa (Khanh et al., 2009). To determine the potential involvement of allelopathic mechanisms of root exudates in $B$. pilosa, photosynthesis in the $P$. multifida gametophyte was investigated. Meanwhile, a non-invasive plant, Coreopsis basalis (Compositae), was selected as a control to determine the possible damage caused by the invasive plant.

\section{Methods}

\section{Plant material}

Root exudate collection of B. pilosa and C. basalis

The seeds of $B$. pilosa and $C$. basalis were rinsed carefully and thoroughly with 
distilled water. Subsequently, the seeds were allowed to germinate in Baltisches Substrat at $25^{\circ} \mathrm{C}$. After 15 days, 40 uniform seedlings $(15 \mathrm{~cm}$ tall) from each plant species were selected and washed thoroughly with distilled water. The seedlings were cultivated in a root exudate collection machine with 1/100 strength Hoagland nutrient solution, with a day/night photoperiod of $16 / 8 \mathrm{~h}$, temperature of $25 / 16^{\circ} \mathrm{C}$, and humidity of $80 / 90 \%$. The silica gel column chromatography was connected with the root exudate collection machine, and the solution was allowed to flow at $5 \mathrm{ml} \cdot \mathrm{min}^{-1}$. After continuous collection for 2 days, chromatography was eluted with methanol. The eluent was evaporated, transferred to tubes, and frozen at $-70^{\circ} \mathrm{C}$ for $72 \mathrm{~h}$. The tubes were then put into the Alpha 1-4 LD plus freeze dryer (Marin Christ, Osterode, Germany) to yield powder root exudates. After that, the root exudates from each plant species were stored at $-20^{\circ} \mathrm{C}$ for further analysis.

\section{Fern gametophyte}

Spores of P. multifida were collected from 15-20 fertile fronds with mature but closed sporangia on September 2012 at Nanjing Forestry University. The fronds were unfolded, placed in clean paper bags, and air-dried at room temperature. One week later, the spores were collected and screened using an 0.088-mm diameter mesh (Zhejiang Shangyu Yarn and Sieve Factory, Shangyu, China). Spores were spread evenly in plastic trays (measuring $25 \mathrm{~cm} \times 20 \mathrm{~cm} \times 5 \mathrm{~cm}$ ) with a sieved mixture of dark soil and sand (Zhang et al., 2011) at an average density of 100-150 spores per $\mathrm{cm}^{2}$. The trays were covered with transparent plastic film to avoid contamination and desiccation, with fluorescent light (photon flux density $1000 \mathrm{~mol} \mathrm{~m}{ }^{-2} \cdot \mathrm{s}^{-1}$ ) at $25^{\circ} \mathrm{C}$ for a 12-h light photoperiod. After spore sowing for twenty days, healthy and uniform gametophytes $(10 \times 5 \mathrm{~mm})$ were selected and rinsed carefully and thoroughly with distilled water.

\section{Treatments application}

Root exudates from each plant species were diluted with 1/1000 strength Hoagland 
nutrition solution to produce five concentrations (0, 12.5, 25, 50 and 100\%). The 1/1000 strength Hoagland nutrition solution was used as a control. Afterwards, all gametophytes were treated with $5 \mathrm{~mL}$ of exudates in a Petri dish (60-mm diameter). One hundred gametophytes were grown in each Petri dish with fluorescent light (37.5 $\left.\mathrm{mol} \mathrm{m} \cdot \mathrm{s}^{-1}\right)$ at $25^{\circ} \mathrm{C}$ for $12 \mathrm{~h}$ light photoperiod.

\section{Fluorescence and pigment measurements}

Fluorescence and pigments of fern gametophytes were analyzed every other day until growth for 10 days. The gametophytes were collected immediately before the fluorescence measurement. Samples were dark-adapted for 2 min. Fluorescence measurements were carried out using a Junior PAM (Heinz Walz GmbH, D91090, Effeltrich, Germany). The fluorometer was connected to a PAM Data Acquisition System PDA 100 (Walz) controlled by the software WINCONTROL v3 (Heinz Walz $\mathrm{GmbH})$. Actinic light intensity (PAR) was $66 \mathrm{~mol} \mathrm{~m}^{-2} \cdot \mathrm{s}^{-1}$. PSII $, \mathrm{Fv} / \mathrm{Fm}, \mathrm{qP}$, ETR and NPQ were recorded and calculated by the formulas of Hoegh-Guldberg et al. (1999). The efficiency of dark-adapted PSII was calculated by Fv/Fm $=(\mathrm{Fm}-\mathrm{Fo}) / \mathrm{Fm}$, where Fm and Fo are the maximum and the minimum fluorescence of dark-adapted samples, respectively.

For the pigment measurements, plant samples $(0.025 \mathrm{~g}$ of fresh weight $)$ from each plant species were used. Chlorophyll was extracted with a 5-mL mixture of ethanol and acetone $(1: 1, \mathrm{v} / \mathrm{v})$ in darkness. A colorimetric procedure was performed until the samples were visibly colorless. The optical densities (OD) of the extracts were determined to be 470, 647 and $663 \mathrm{~nm}$ using a SP-2100P spectrophotometer (Spectroscopic Instrument Inc., Shanghai, China). Subsequently, the optical densities were converted to chlorophyll $a(\mathrm{Chl} a)$, chlorophyll $b(\mathrm{Chl} b)$, carotenoid (Car), and total chlorophyll content following the formulas of Moran (1982). 
One-way analysis of variance (ANOVA) was conducted on photosynthesis with exudate treatments for each plant species. Descriptive statistics were used to analyze central tendency and dispersion trends of the data. Mean differences were separated using least-squares difference (LSD) and Duncan's test with $\mathrm{P}=0.05$ indicating statistical significance. The comparative effect between invasive and non-invasive plant species on the fluorescence of the gametophyte was calculated by t-test for paired samples at $\mathrm{P}=0.05$. Statistical analyses were performed by using SPSS v.20.0 for Windows (IBM; Armonk, New York, USA); all graphs were drawn using Origin 8.0 (Origin Lab, Northampton, Massachusetts, USA). The detail data on the comparative effects between B. pilosa and C. basalis on PSII (A), Fv/Fm (B), qP (C), ETR (D) and NPQ (E) in the gametophyte described by t-test for paired samples is presented in Table 1, which is available online as Supplementary material in electronic copy of the journal as well as on our server because of the repetition with figures.

Results

Five fluorescence indexes of photosystem II (PS II) of the gametophytes were recorded with exposure of the plant samples to B. pilosa and C. basalis exudates. The chlorophyll pigments of the gametophytes were measured under the treatments of root exudates, and all the data were collected on days $2,4,6,8$, and 10 of the experiment.

PSII

PSII is an important index about the photosynthetic abilities of a given plant; the index reflects the ratio between the yield of PS II photochemical quantum and total photochemical quantum. The ${ }_{\text {PSII }}$ of $P$. multifida decreased with increasing root exudate concentrations and exposure time after gametophytes were exposed to both $B$. pilosa and C. basalis exudates (Fig. 1A, B). For exposure of the $100 \%$ root exudate of B. pilosa at day 10, the ${ }_{\text {PSII }}$ of the gametophyte reached the minimum at 0.10 (Fig. 1A). 
In comparison with the control, PsII of $P$. multifida did not markedly decrease at day 2 with exposure to $C$. basalis, while ${ }_{\text {PSII }}$ predominantly decreased during days 4-10. PSII was reduced to $27.5 \%$ at day 10 after gametophytes were exposed to $100 \%$ root exudate of $C$. basalis (Fig. 1B). In comparison with $C$. basalis exudate, after the application of $B$. pilosa, no significant difference in ${ }_{\text {PSII }}$ of $P$. multifida was noted at day 2 at a $12.5 \%$ concentration (Table 1A). Afterwards, PSII differed significantly from day 4 to day 10 between the treatments of B. pilosa and C. basalis. Meanwhile, Table 1 shows that the changes in ${ }_{\text {PSII }}$ of the gametophytes were significantly different after exposure to $B$. pilosa and $C$. basalis exudates at a concentration of $25 \%$ or above from day 2 to day 10 .

\section{$F v / F m$}

$\mathrm{Fv} / \mathrm{Fm}$ is an index to estimate the maximum portion of absorbed quanta used in PSII reaction centers. Fv/Fm of $P$. multifida was also affected by the root exudate of $B$. pilosa (Fig. 2A), gradually decreasing with the addition of root exudates. Fv/Fm leveled off in the control, while remarkable decreases were found at days 2-10 at all levels of root exudate exposure. In the control, Fv/Fm values were 0.70-0.69. However, Fv/Fm decreased to 0.52 at day 2 and 0.22 at day 10 under $12.5 \%$ treatments (Fig. 2A). Fv/Fm of $P$. multifida decreased with the increase of root exudate concentrations of C. basalis (Fig. 2B). Fv/Fm remarkably decreased during days 4-10 except for $12.5 \%$ treatment at day 4 , compared with the control. Fv/Fm values were 0.77 and 0.68 in the control at day 2 and $100 \%$ treatment at day 10, respectively (Fig. 2B). The data points from the samples treated with B. pilosa and C. basalis exudates demonstrated differences with statistical significance (Table 1B).

$q P$

$\mathrm{qP}$ allows the quantification of both the effective photochemical state of the PSII regarding the fraction of PSII centers that remain open or oxidized at any time, and 
the non-photochemical photosynthetic mechanisms involved in photo-protection, state 1 and state 2 transition quenching, photo-inhibition, and photo-damage. The inhibitory effect of $B$. pilosa exudates on the $\mathrm{qP}$ of gametophytes increased with increasing concentrations, indicating a negative dose-dependent effect between the exudate concentrations and $\mathrm{qP}$ (Fig. 3A). The differences were significant in inhibitory effects on gametophyte of $P$. multifida between the exudate treatments and the control. qP decreased by $81.3 \%$ after the gametophyte was exposed to $100 \% \mathrm{~B}$. pilosa exudate for 10 days (Fig. 3A). The presence of high concentrations (especially a $100 \%$ concentration) of $C$. basalis exudates resulted in significant inhibition for the qP of the gametophytes (Fig. 3B). The average inhibitory rate was $67.9 \%$ for day 8 with the application of $100 \%$ root exudate. $\mathrm{qP}$ decreased by $35.7 \%$ upon exposure to $100 \%$ C. basalis exudate for 10 days. Comparative inhibition between B. pilosa and C. basalis of the qP of P. multifida followed a similar pattern as with ${ }_{\mathrm{PSII}}$ (Table 1C).

\section{Electron transport rate (ETR)}

Electron transport rate (or ETR) is valuable for many types of plant stress investigations. In this study, the variation in ETR was similar to that of qP, decreasing with the increase of root exudate concentrations of both species (Fig. 4A, B). Plants had their lowest ETR level $\left(2.10 \mu \mathrm{mol} \cdot \mathrm{m}^{-2} \cdot \mathrm{s}^{-1}\right)$ when treated with $100 \%$ B. pilosa exudate at day 10 . ETR decreased by $81.1 \%$ compared to the control with increases in $B$. pilosa exudate concentration to $100 \%$. The highest ETR was $10.10 \mu \mathrm{mol} \cdot \mathrm{m}^{-2} \cdot \mathrm{s}^{-1}$ at day 4 with $12.5 \%$ B. pilosa exudates in comparison with the higher treatments (Fig. 4A). However, ETR remarkably decreased at days 6 and 10 in various concentrations of $C$. basalis exudates (Fig. 4B). Generally, B. pilosa exudates significantly decreased ETR compared with $C$. basalis exudates, except in the case of $12.5 \%$ concentration, day 2 (Table 1D).

Non-photochemical quenching $(N P Q)$ 
Non-photochemical quenching (NPQ) is more heavily affected in instances where NPQ reflects heat-dissipation of excitation energy in the antenna system. The NPQ of P. multifida was enhanced with increasing root exudate levels of B. pilosa and $C$. basalis (Fig. 5). Compared to the control, application of $12.5 \%$ root exudate of $B$. pilosa resulted in 1.14- to 1.55-fold increases in NPQ at days 2 and 10, respectively (Fig. 5A). The treatment of $100 \%$ root exudate of B. pilosa induced a 2 -fold increase in NPQ at day 2 as well as a 2.45-fold increase at day 10 compared to the control. With regard to the treatments of $C$. basalis, the NPQ of $P$. multifida increased from 0 to $33.3 \%$ during the experimental period (Fig. 5B). In particular, NPQ was remarkably affected by increased root exudates of $C$. basalis at day 6. Fig. 5B showed that NPQ increased from 0.24 to 0.28 at $100 \%$ concentrations for $C$. basalis from days 2 to 10 . A comparative influence between $B$. pilosa and C. basalis on NPQ of P. multifida followed a similar pattern as ETR (Table 1E).

\section{Pigments of gametophytes}

Chl $a$, Chl $b$, Car, and total chlorophyll content decreased as root exudate concentrations increased (Fig. 6A-D). This change was also reflected by the change in color of the gametophyte. When the applied root exudate increased from $12.5 \%$ to $25 \%$, Chl $a$ and Chl $b$ content at day 10 were reduced by $18.85 \%$ and $12.50 \%$, respectively $(P<0.05)$. Surprisingly, the changes of Chl $a$ and Chl $b$ were almost similar in samples treated with $25 \%$ and $50 \%$ root exudates (Fig. 6A-B).

Car content with $100 \%$ root exudate treatment exhibited the lowest levels compared to the control. The values of Car at day 2 and 10 with the addition of $100 \%$ root exudate were 0.19 and $0.16 \mathrm{mg} / \mathrm{g}$, for a $33.34 \%$ and $44.83 \%$ reduction in comparison with the control (Fig. 6C). Treatment of $12.5 \%$ root exudate resulted in a greater decline in the total chlorophyll than other treatments (Fig. 6D). At day 2 and 10, $12.4 \%$ and $20 \%$ reductions in total chlorophyll were observed with the application of $12.5 \%$ root exudate compared with the control. In contrast, the total chlorophyll 
decreased by $6.01 \%$ and $4.32 \%$ when root exudate concentration was increased from $25 \%$ to $50 \%$ (Fig. 6D).

\section{Discussion}

According to the results, the five fluorescence indexes and the pigments demonstrate a response with increases in the root exudates concentrations. In addition, the inhibitory effects on the chlorophyll fluorescence in gametophytes caused by $B$. pilosa root exudates are more significant and stronger than those caused by $C$. basalis. The environmental stress would enhance the extent of photo-inhibition, and the process is determined by the balance between the rate of photo-damage to PSII and the rate of its repair (Murata and Takahashi, 2008). It is found that Fv/Fm, PSII, $\mathrm{qP}$, and ETR are markedly decreased in the presence of $B$. pilosa exudates at a concentration of $12.5 \%$ or above. The value of NPQ increases at various concentrations.

In this study, with the decrease of $\mathrm{Fv} / \mathrm{Fm}$ and ${ }_{\mathrm{PSII}}$, the absorbed B. pilosa root exudates in the gametophyte significantly damage the production of quantum yield and PS II photochemical quantum yield, as well as the relative quantum yield in the PS II process, with the ETR in PS II also declining. We assume that the decrease of photochemical quantum production and its yield retard the energy conversion from photon to electron, while the protective system also weakens with increasing concentrations of root exudates. This trend seems to endure and is not reversible, according to the results of the $\mathrm{qP}$. In photosynthesis reaction centers, the production of quantum yield results in the fading of PS II, with subsequent decreases in ETR and the relative photochemical quantum yield. However, $C$. basalis root exudates cause little damage on the photosynthetic ETR of gametophyte, the trends of decline for Fv/Fm, PsII, qP, and ETR seem smooth and steady in the treatments. These decreases are not significant at the same treatment, with the NPQ not having a significant increase as a result. The results clarify that the damage caused by invasive root exudates is more harmful than that from native plant species, especially with respect 
to the photosynthetic electron-transfer reaction.

In addition, the pigments ( $\mathrm{Chl} \mathrm{a}, \mathrm{Chl} \mathrm{b}$ and $\mathrm{Car})$ also decline with the increasing root-exudate concentrations, especially $\mathrm{Chl} \mathrm{a}$ and $\mathrm{Chl} \mathrm{b}$, which are important parts in photosystems I and II (PS I and PS II). It can be inferred that the components of root exudates also have the function of promoting degradation or hindering of the synthesis of pigments. Photosystems I and II are the two major photosynthetic units, and they are mainly distributed at the non-stack battlements stroma and grana lamellae and stack battlements interstitial in the thylakoid membrane in chloroplasts. The pigments in chloroplasts have the functions of receiving, transferring, and converting energy in photosynthesis. In PS I, Chl a is the primary electron donor P700 and the original acceptor of the P700 excited state (Plato et al., 2003). In PS II, the chloroplast pigments $\mathrm{Chl} \mathrm{a,} \mathrm{Chl} \mathrm{b,} \mathrm{and} \mathrm{Car} \mathrm{play} \mathrm{an} \mathrm{important} \mathrm{role} \mathrm{in} \mathrm{light} \mathrm{harvesting}$ and energy transport (Leeuwen et al., 1991). In this study, the decrease of Chl a, Chl b, and Car in the presence of B. pilosa root exudates inhibits light harvesting and slows ETR in PS I and II. The concentration of pigments decreases due to allelochemicals, which leads to the degradation or depression of pigment synthesis under treatment.

Figure 7 provides the improved schema under root exudate treatment according to the Z-schema (zigzag schema) of the photosynthetic electron-transfer reaction chain. With the increasing effects of root exudates, some changes occur in PS I and II due to this stress. The electronic and energy chain transferring from PS II to PS I is caused by an oxidation-reduction reaction; the plant can use the light to produce chemical energy, and the chain is more reliant on the electron-transfer reactions in chloroplasts. $\mathrm{H}_{2} \mathrm{O}$ is oxidized in $\mathrm{O}_{2}$, and the electron is transferred to the PS II reaction center. With the energy of photon P680, the ground state of the accepter is excited enough to convert electric energy. P680 is mainly made up by $\mathrm{Chl}$ a, $\mathrm{Chl} \mathrm{b}$ and Car, and these three pigments form a chain from accepter to donor, and this chain gives the electron to PS I through a series of compounds-PQ-PQH ${ }_{2}$ (plastoquinones), Cybt6f (cytochrome complex), and PC (plastocyanin). Under the root-exudate treatments, the effective quantum yields in PS II reaction centers are limited, with the negative effects 
resulting in reductions of the oxidation reaction. Meanwhile, this study reveals that the electron transport from PS II to PS I is interrupted as the effective excited electron decreases with the effects caused by root exudates on pigments. The result indicates that too much photonic energy is dissipated in a non-regulated manner (such as heat or uncoupling), as the method of regulation is interrupted. Weakened electron-transfer reaction is the main inhibition effect caused by $B$. pilosa, and reduction in the pigments of the gametophyte, $\mathrm{Chl} \mathrm{a,} \mathrm{and} \mathrm{Chl} \mathrm{b}$ are a decisive reason for ETR, the photon receive and electron transport. Decreases in the pigments of native species caused by $B$. pilosa root exudates are the primary cause of damage to the photosynthetic electron-transfer reaction.

It is well reported that extracts from Ephedra equisetina root are capable of inducing destruction of the thylakoid membrane, interruption of electron transport, and reduction of effective quantum yield, thus causing cessation of photosynthesis and inducing cyanobacterial death (Yan et al., 2012). In PS I, the system could use the electron from PS II and the photon to reduce $\mathrm{NADP}^{+}$(triphosphopyridine nucleotide), and store the energy in NADPH. The conversion is primarily based on the excitatory process of P700 so that the reaction center is concerned with $\mathrm{Chl}$ a, and the fluorescence indexes are applied to reflect this reaction. The chlorophyll fluorescence of $P$. multifida was adversely affected by $B$. pilosa root exudates, which is similar to the description of the chlorophyll fluorescence analysis of photosynthetic efficiency, quantum yield, and photon energy dissipation in photosynthesis antennae of Lactuca sativa leaves exposed to cinnamic acid given by Hussain and Reigosa (Hussain and Reigosa, 2011b). The reduced sensitivity of Fv/Fm to B. pilosa root exudates was due to the conserved $\mathrm{F}_{0}$ in relation to lower chlorophyll content, indicating a low-level functioning of PS II with a low absorption rate of light in the gametophytes. The results for the chlorophyll contents are in line with those from preliminary studies on Chlorella vulgaris by Qian et al. (2009) and Microcystis aeruginosa NIES-843 (cyanobacteria) by Shao et al. (2011). Our results show that the contents of chlorophyll and Car decreased upon the exposure of B. pilosa root exudates. In this 
study, we find that the effects of $B$. pilosa root exudates could cause damage to photosynthesis, both on PS I to PS II, and the components of the root exudates have the enduring and irreversible destruction on the energy transformation from photon to electron, and from electron to chemical energy in plants.

It is reported that the photosystem is more sensitive to herbicides, and the organics are harmful to the cyclic electron flow from PS I center to PS II center and the energy conversion from electron to chemicals, such as DCMU (3-(3,4-dichlorophenyl)-1,1-dimethylurea) and Gramoxone (Hosler and Yocum 1987; Allen and Nilsson 1997). However, the natural organics, such as the root exudates in this study, play a different role in the destruction of the photosystem. The organics affect photosynthesis by degrading the pigments; loss of pigments leads to photon acceptance, electron transport, and energy conversion. Chlorophyll plays an important role in the absorption, transfer, and transformation of light energy. Meanwhile, Car is the indispensable component of chromoprotein in photosynthesis, which is important for the protection of photosynthetic apparatus from injury caused by the presence of singlet oxygen. Decreases in Car block photon transfer in PS II, with the additional weakness of the overall antioxidant effect. The fluorescence indexes given in this study demonstrate that the root exudates have considerable damaging effects on PS I and PS II. The loss of pigments is the main reason for the destructive effects on the photosystem (caused by the allelochemicals) and the root exudates.

The donor invasive plant species could have fatal, weak or non-allelopathic effects on several target species, which belong to different taxonomic families. As a result, small but important differences are expected in their physiology associated with the allelochemical mode of action (Lorenzo et al., 2011). The observed loss of fern species in B. pilosa patches in the field, together with the results of our laboratory test, implies that the effects of $B$. pilosa on native species are deleterious and allelopathy may be partly responsible for the adverse effects. Meanwhile, in comparison with $B$. pilosa exudates, PSII, Fv/Fm, qP, and ETR in P. multifida decreased slightly in $C$. basalis exudate treatments. The present comparative research on $B$. pilosa and $C$. 
basalis reveals that $B$. pilosa is the invasive plant species responsible for the gametophyte damage.

The Novel Weapons Hypothesis set to explain the remarkable success of many exotic invasive species argues that invaders may possess novel chemicals that are more phytotoxic to plants in the invaded range than to adapted species in the invader's native range (Zhu et al., 2011). With regard to B. pilosa, many secondary metabolites (phenolics, polyacetylenes, and triterpenes) involved in allelopathic action were found; of these, phenylheptatriyne (PHT) and its derivatives amounts were highest in $B$. pilosa oil (Khanh et al., 2009). A total of 23 phenolic compounds (including salicylic acid, vanillin, p-hydroxybenzoic acid, p-coumaric acid, and ferulic acid) were identified and isolated in its shoots and roots. Contents of caffeic acid were highest (117.4, 298.7, and $350.3 \mathrm{~g} \mathrm{~g}^{-1}$ in leaves, stems and roots), followed by pyrocatechin (Khanh et al., 2009).

The detection and study of allelochemicals in B. pilosa root exudates, which are responsible for inhibition of $P$. multifida gametophyte, continue at our laboratory. About 30 allelochemicals were detected in B. pilosa root exudates (data not shown). Isolation, identification, and characterization of phytotoxic substances in B. pilosa root exudates should be further investigated. Determination of the mechanism and modes of action of these allelochemicals should be one area of particular focus. Moreover, allelopathic research of the impact of $B$. pilosa root exudates on other physiological and primary processes (except for photosynthesis) should be carried out, since root exudate of donor plant species can affect the receptor through several different mechanisms of action (Dayan et al., 2009). Besides, the effects of the stem, leaf, and flower of B. pilosa on the P. multifida gametophyte should be addressed due to the possible variety and concentrations of allelochemicals in different parts of plants. Although we are still in the early stages of understanding the gametophyte responses of $P$. multifida to the root exudates of B. pilosa, this study opens the door to understanding of the photosynthetic response of the fern to invasive plant species. 


\section{Conclusions}

It is concluded that $B$. pilosa enduringly and irreversibly affects the photosynthesis of P. multifida via allelopathic interference, both on PS I and PS II. Decreases in the acceptors of light and electron transport in PS reaction centers and the production of photochemical quantum yield in PS II are the main factors of this that are precipitated by the stress caused by root exudates. This comparative research study of invasive and non-invasive plants in allelopathic experiments demonstrated that invasive plants are responsible for the critical damage to this local species.

\section{Acknowledgements}

This work was supported by the National Natural Science Foundation of China (31200233), the Priority Academic Program Development of Jiangsu Higher Education Institutions (PAPD), Collaborative Innovation Plan of Jiangsu Higher Education, the Jiangsu Government Scholarship for Overseas Studies and the Laboratories of Analytical Biology of the National Museum of Natural History, the Smithsonian Institution.

\section{References}

Allen, J.F., Nilsson, A. 1997. Redox signalling and the structural basis of regulation of photosynthesis by protein phosphorylation. Physiologia Plantarum 100(4):863-868.

Ashraf M., Foolad M.R. 2007. Roles of glycine betaine and proline in improving plant abiotic stress resistance. Environmental and Experimental Botany 59(2): 206-216.

Bais, H.P., Vepachedu, R., Gilroy, S., Callaway, R.M., Vivanco, J.M. 2003. Allelopathy and exotic plant invasion: from molecules and genes to species interactions. Science 301, 1377-1380. 
Dayan, F.E., Howell. J.L., Weidenhamer, J.D. 2009. Dynamic root exudation of sorgoleone and itsin planta mechanism of action. Journal of Experimental Botany $60,2107-2117$.

de Groot, G.A., During, H.J., Maas, J.W., Schneider, H., Vogel, J.C., Erkens, R. H. J. 2011. Use of rbcLand trnL-F as a two-locus DNA barcode for identification of NW-European ferns: an ecological perspective. Plos one 6, e16371. doi: 10.1371/journal.pone.0016371.

Hao, J.H., Liu, Q.Q., Qiang, S. 2009. Reproductive traits associated with invasiveness in Bidens pilosa. Chinese Bulletin of Botany. 44, 656-665.

Hoegh-Guldberg, O., Jones, R.J. 1999. Photoinhibition and photoprotection in symbiotic dinoflagellates from reef-building corals. Marine Ecology Progress Series $183,73-86$.

Hosler, J.P., Yocum, C.F. 1987. Regulation of Cyclic Photophosphorylation during Ferredoxin-Mediated Electron Transport : Effect of DCMU and the NADPH/NADP Ratio. Plant Physiology 83(4):965-969.

Hussain, M.I., Reigosa, M.J. 2011a. Allelochemical stress inhibits growth, leaf water relations, PSII photochemistry, non-photochemical fluorescence quenching, and heat energy dissipation in three $\mathrm{C}_{3}$ perennial species. Journal of Experimental Botany 62, 4533-4545.

Hussain, M.I., Reigosa, M.J. 2011b. A chlorophyll fluorescence analysis of photosynthetic efficiency, quantum yield and photon energy dissipation in PSII antennae of Lactuca sativa L. leaves exposed to cinnamic acid. Plant Physiology and Biochemistry 49, 1290-1298.

Khanh, T.D., Cong, L.C., Xuan, T.D., Uezato, Y., Deba, F., Toyama, T., Tawata, S. 2009. Allelopathic plants: 20. hairy beggarticks (Bidens pilosa L.). Allelopathy Journal 24, 243-254.

Leeuwen, P.J., Nieveen, M.C., Meent, E.J., Dekker, J.P., Vangorkom, H.J. 1991.Rapid and simple isolation of pure photosystem II core and reaction center particles from spinach. Photosynthesis Research 28(3):149-153. 
Liu, Y., Shen, Y., Zhang, K.M., Fang, Y.M., Yang, J. 2015. Effects of root exudates of Bidens pilosa on growth and development of gametophyte of Pteris multifida. Acta Agriculturae Boreali-occidentalis Sinica 24: 149-155.

Lorenzo, P., Palomera-Pérez, A., Reigosa, M.J., González, L. 2011. Allelopathic interference of invasive Acacia dealbata Link on the physiological parameters of native understory species. Plant Ecology 212, 403-412.

Lu, J.M., Zhang, N., Du, X.Y., Wen, J., Li, D.Z. 2015. Chloroplast phylogenomics resolves key relationships in ferns. Journal of Systematics and Evolution 53, 448-457.

Moran, R. 1982. Formulae for determination of chlorophyllous pigments extracted with N, N-dimethylformamide. Plant physiology 69, 1376-1381.

Murata, N., Takahashi, S. 2008. How do environmental stresses accelerate photoinhibition?. Trends in Plant Science 13(4): 178-182.

Parker, Jr. E.D., Forbes, V.E., Nielsen S.L., Ritter C., Barata C., Baird D. J. 1999. Admiraal W., Levin L., Loeschke V., Lyytikäinen-Saarenmaa P., Høgh-Jensen H., Calow P., Ripley B. J. Stress in Ecological Systems. Oikos 86(1): 179-184.

Plato, M., Krauß, N., Fromme, P., Lubitz, W. 2003. Molecular orbital study of the primary electron donor P700 of photosystem I based on a recent X-ray single crystal structure analysis. Chemical Physics 294(3):483-499.

Pimentel, D., Zuniga, R., Morrison, D. 2005. Update on the environmental and economic costs associated with alien-invasive species in the United States. Ecological Economics 52, 273-288.

Qian, H., Xu, X., Chen, W., Jiang, H., Jin, Y., Liu, W., Fu, Z. 2009. Allelochemical stress causes oxidative damage and inhibition of photosynthesis in Chlorella vulgaris. Chemosphere 75, 368-375.

Raj, A., Pandey, A.K., Sharma, Y.K., Khare, P.B., Srivastava, P.K., Singh, N. 2011. Metabolic adaptation of Pteris vittata L. gametophyte to arsenic induced oxidative stress. Bioresource technology 102, 9827-9832. 
Ruan, X., Li, Z.H., Wang, Q., Pan, C.D., Jiang, D.A., Wang, G.G. 2011. Autotoxicity and allelopathy of 3, 4-dihydroxyacetophenone isolated from Picea schrenkiana needles. Molecules 16, 8874-8893.

Shao, J., Xu, Y., Wang, Z., Jiang, Y., Yu, G., Peng, X., Li, R. 2011. Elucidating the toxicity targets of -ionone on photosynthetic system of Microcystis aeruginosa NIES-843 (Cyanobacteria). Aquatic Toxicology 104, 48-55.

Vitousek, P.M., D’ Antonio, C.M., Loope, L.L., Westbrooks, R. 1996. Biological invasions as global environmental change. American Scientist 84(5): 218-228.

Wang W., Vinocur B., Altman A. 2003. Plant responses to drought, salinity and extreme temperatures: towards genetic engineering for stress tolerance. Planta 218(1): 1-14.

Weir, T.L., Park, S.W., Vivanco, J.M. 2004. Biochemical and physiological mechanisms mediated by allelochemicals. Current Opinion in Plant Biology 7, 472-479.

Yan, R., Ji, H.L., Wu, Y.H., Kerr, P.G., Fang, Y.M., Yang, L.Z. 2012. An investigation into the kinetics and mechanism of the removal of Cyanobacteria by extract of Ephedra equisetina root. PloS One 7, e42285.

Zhang, K.M., Shen, Y; Fang, Y.M. 2015. Changes in the gametophyte physiology of Pteris multifida induced by Bidens Pilosa leaves. Environmental Science and Pollution Research. doi: 10.1007/s11356-015-5589-x.

Zhang, K.M., Shi, L., Jiang, C.D., Li, Z.Y. 2007. Influence of root, stem and leaf leachates of Ageratina adenophora weed on the gametophyte development of Pteris finotii. Allelopathy Journal 20, 203-212.

Zhang, K.M., Shi, L., Jiang, C.D., Li, Z.Y. 2008a. Inhibition of Ageratina adenophora on spore germination and gametophyte development of Macrothelypteris torresiana. Journal of Integrative Plant Biology 50, 559-564.

Zhang, K.M., Shi, L., Jiang, C.D., Li, Z.Y. 2008b. Allelopathic effects of Eupatorium adenophorum on spore germination and gametophyte development in Cibotium barometz. Acta Pratacult Urae Sinica 17, 19-25. 
Zhang, K.M., Liu, B.D., Fang, Y.M., Shi, L., Tang, S.J. 2011. Studies on the gametophytes of eight Chinese species of Dryopteris (Dryopteridaceae). American Fern Journal 101, 12-24.

Zhang, K.M., Liu, J.H., Cheng, X., Zhang, G.F., Fang, Y.M., Zhang, H.J. 2012. Effects of Ageratina adenophora on spore germination and gametophyte development of Neocheiropteris palmatopedata. American Fern Journal 102, 208-215.

Zhu, X.Z., Zhang, J.T., Ma, K.P. 2011. Soil biota reduce allelopathic effects of the invasive Eupatorium adenophorum. Plos One 6, e25393. 
Additional files:

Additional file 1: Figure 1. Impacts of time and root exudations of Bidens pilosa (A) and Coreopsis basalis (B) on quantum yield of photosystem II ( PSII) of Pteris multifida gametophytes. Statistically significant differences among treatments are indicated by different letters $(P<0.05)$.

Additional file 2: Figure 2. Comparison of results of the lower quantum efficiency of open PSII reaction centers $(\mathrm{Fv} / \mathrm{Fm})$ between the gametophyte treated with Bidens pilosa (A) and Coreopsis basalis (B) exudations. Statistically significant differences among treatments are indicated by different letters $(P<0.05)$.

Additional file 3: Figure 3. The reduction of photochemical fluorescence quenching (qP) using Bidens pilosa (A) and Coreopsis basalis (B) exudations. Statistically significant differences among treatments are indicated by different letters $(P<0.05)$.

Additional file 4: Figure 4. Electron transport rate (ETR) of Pteris multifida gametophytes in the treatment groups of Bidens pilosa (A) and Coreopsis basalis (B) exudations. Statistically significant differences among treatments are indicated by different letters $(P<0.05)$.

Additional file 5: Figure 5. Change of non-photochemical quenching (NPQ) of Pteris multifida gametophytes when exposed to Bidens pilosa (A) and Coreopsis basalis (B) exudations. Statistically significant differences among treatments are indicated by different letters $(P<0.05)$.

Additional file 6: Figure 6. The values of A) Chlorophyll $a$, B) Chlorophyll $b, \mathrm{C}$ ) carotenoid and D) the total chlorophyll in the control and treated with B. pilosa root exudations. Statistically significant differences among treatments are indicated by different letters $(P<0.05)$. 
Additional file 7: Figure 7. The improved Z-schema (Zigzag schema) of photosynthetic electron transport chain in fern under Bidens pilosa root exudations. (P680 and P700: chlorophyll in reaction centers) 
Figures

Figure 1
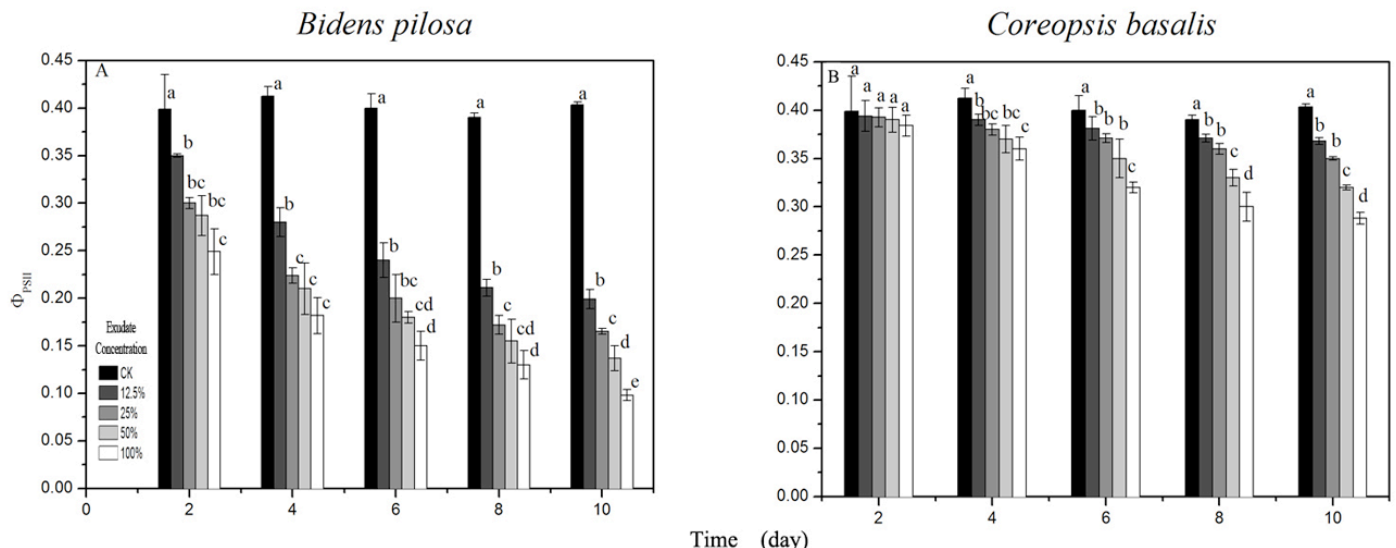

Figure 2
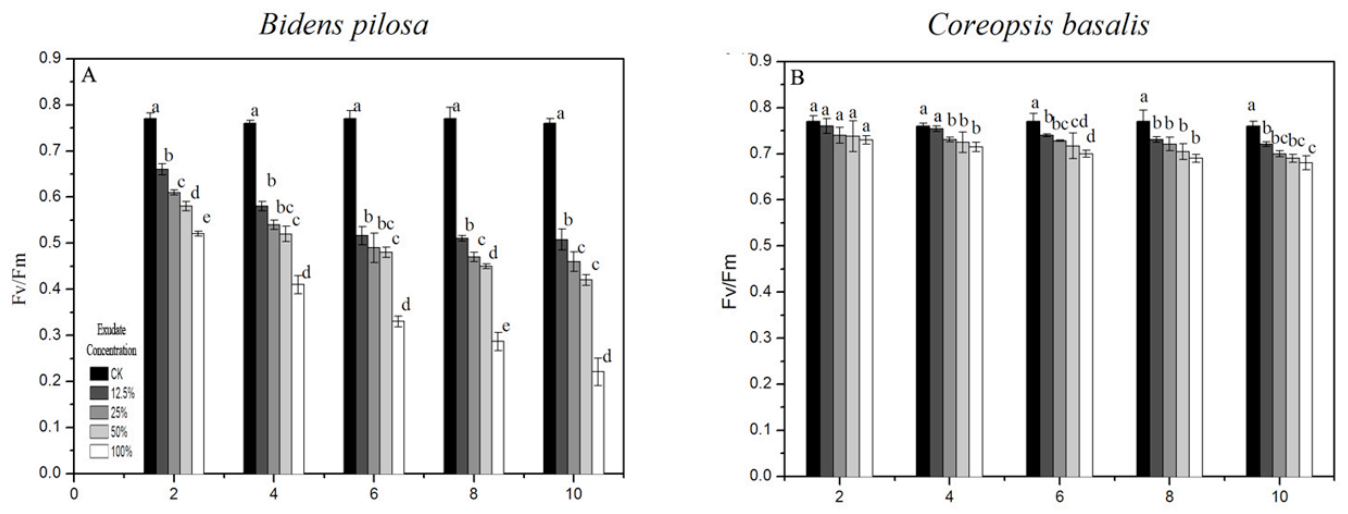

Time (day) 
Figure 3
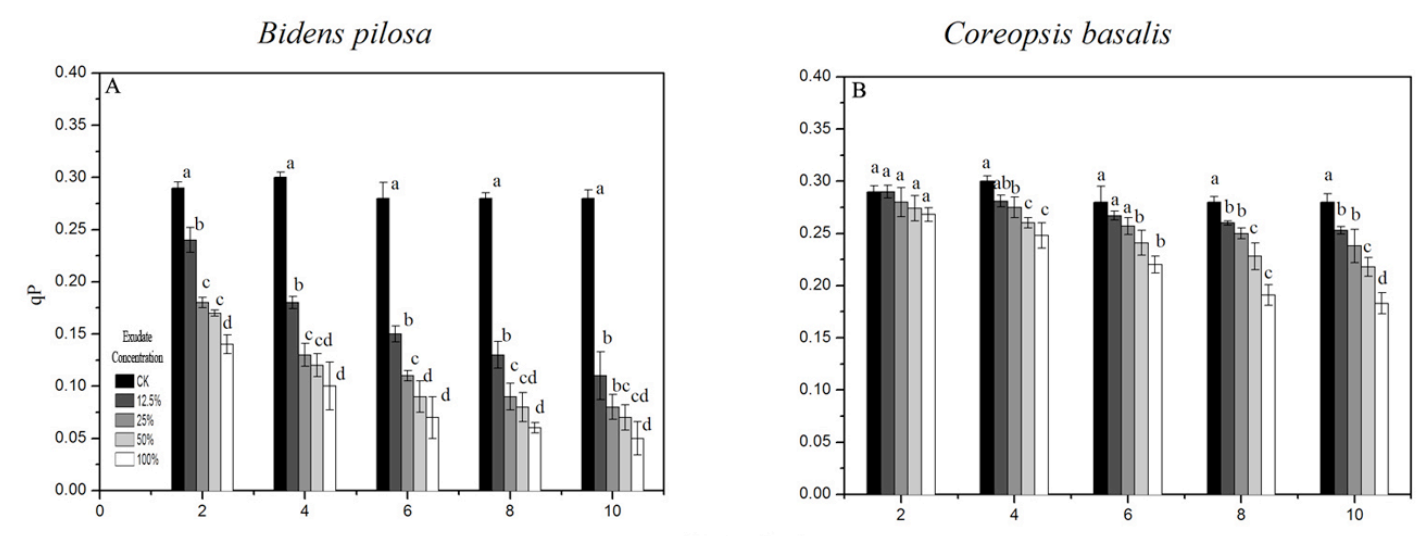

Figure 4
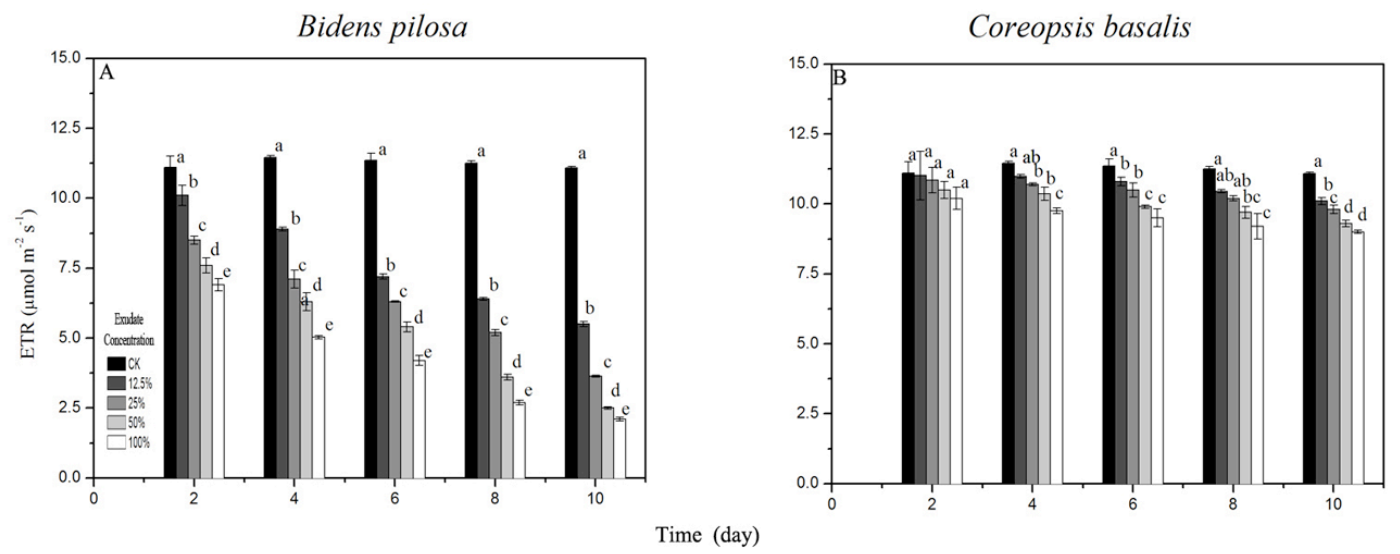
Figure 5

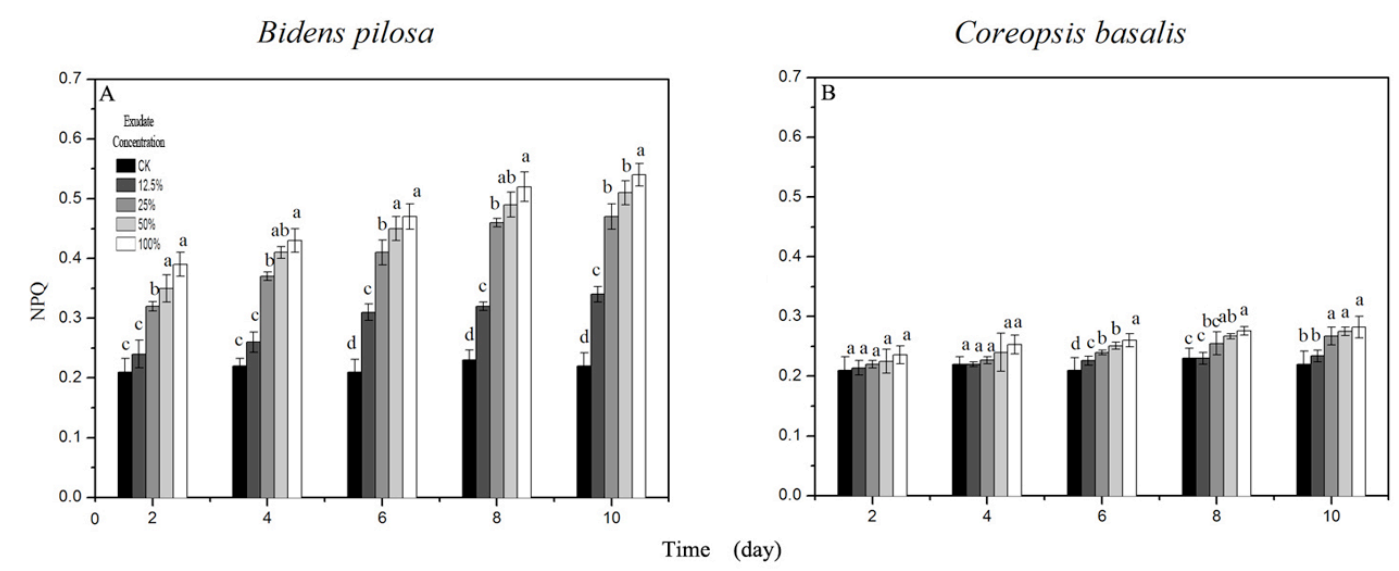

Figure 6
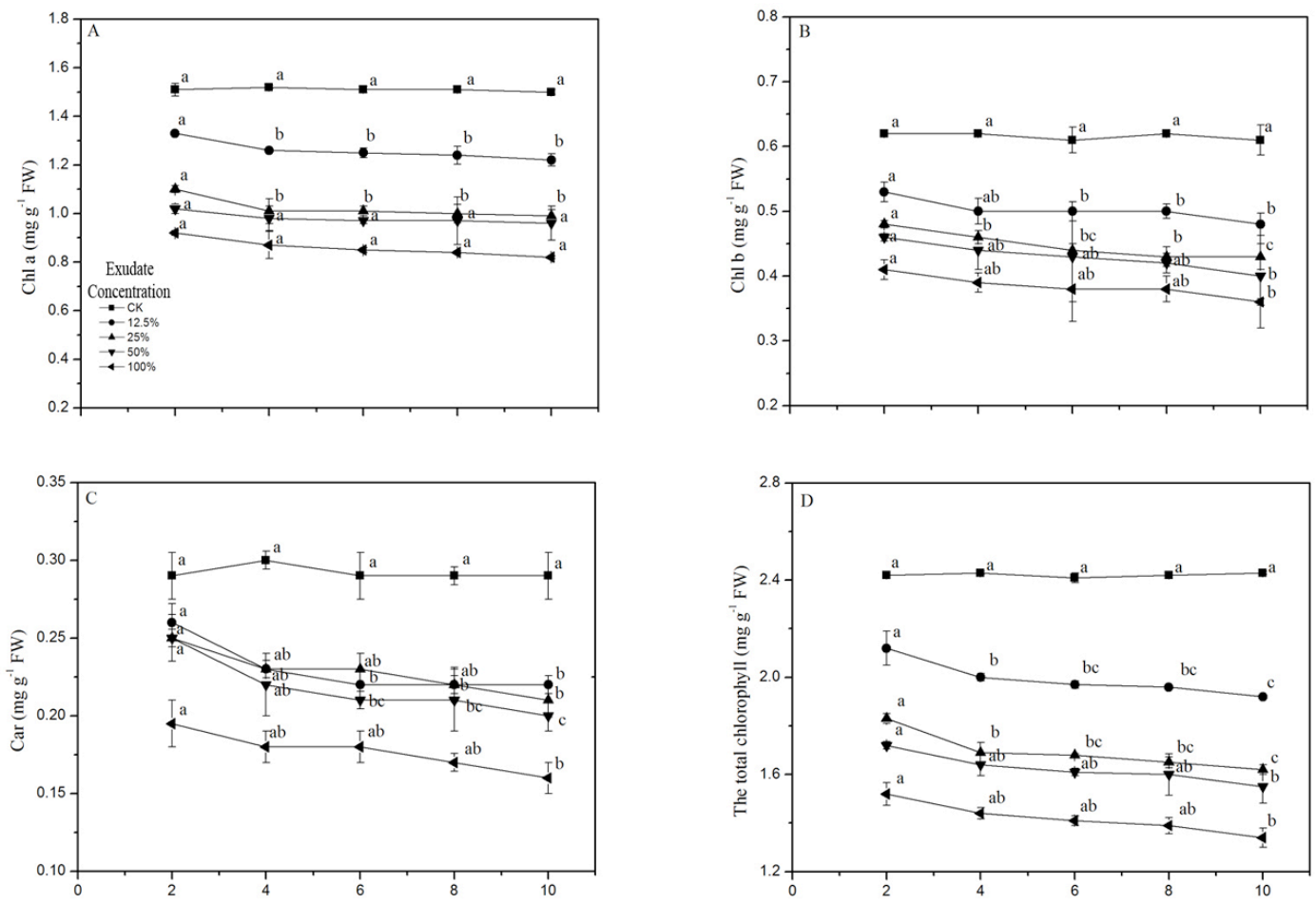

Time (day) 
Figure 7

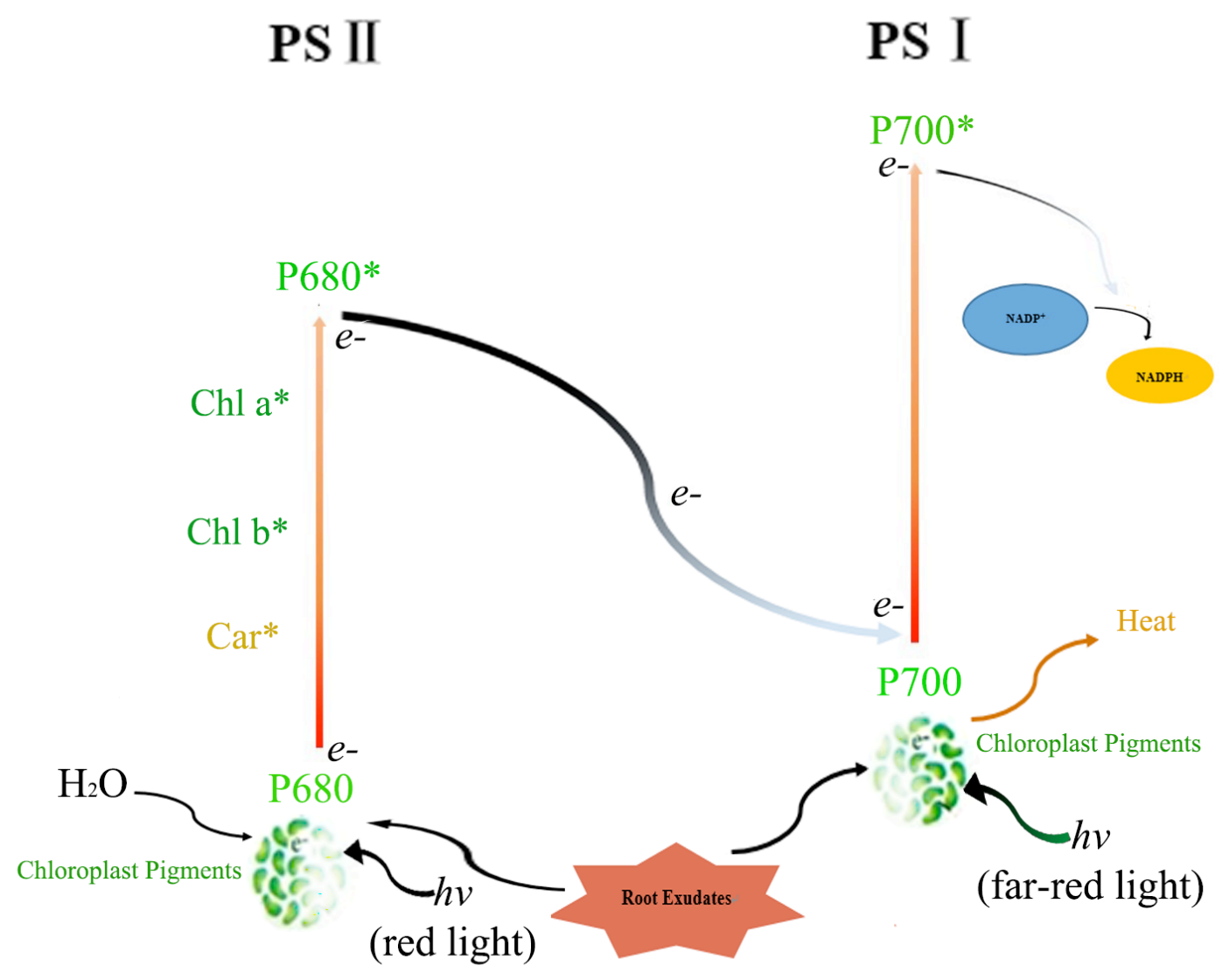

